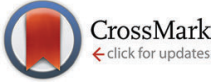

Cite this: Phys. Chem. Chem. Phys., 2016, 18, 6247

Received 1st January 2016, Accepted 24th January 2016

DOI: $10.1039 / \mathrm{c} 6 \mathrm{cp} 00011 \mathrm{~h}$

www.rsc.org/pccp

\title{
Large negative thermal expansion in non-perovskite lead-free ferroelectric $\mathrm{Sn}_{2} \mathrm{P}_{2} \mathrm{~S}_{6} \dagger$
}

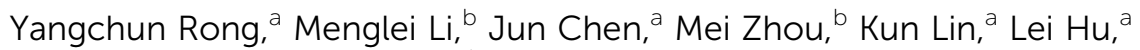 \\ Wenxia Yuan, ${ }^{\mathrm{C}}$ Wenhui Duan, ${ }^{\mathrm{b}}$ Jinxia Deng ${ }^{\mathrm{c}}$ and Xianran Xing*a
}

\begin{abstract}
Functional materials showing both negative thermal expansion (NTE) and physical performance, such as ferroelectricity and magnetism, have been extensively explored in the past decade. However, among ferroelectrics a remarkable NTE was only found in perovskite-type $\mathrm{PbTiO}_{3}$-based compounds. In this work, a large NTE of $-4.7 \times 10^{-5} \mathrm{~K}^{-1}$ is obtained in the non-perovskite lead-free ferroelectric $\mathrm{Sn}_{2} \mathrm{P}_{2} \mathrm{~S}_{6}$ from $243 \mathrm{~K}$ to $T_{\mathrm{C}}(338 \mathrm{~K})$. Structure refinements and first-principle calculations reveal the effects of the $\mathrm{Sn}(I) \mathrm{s}-\mathrm{S} 3 \mathrm{p}$ interaction on spontaneous polarization and its correlation with NTE. Then the mechanism of spontaneous volume ferroelectrostriction (SVFS) is verified and it could well elucidate the nature of NTE in ferroelectric $\mathrm{Sn}_{2} \mathrm{P}_{2} \mathrm{~S}_{6}$. This is the first case to demonstrate the unusual NTE behavior by SVFS in a non-perovskite lead-free ferroelectric material.
\end{abstract}

\section{Introduction}

As an intriguing property of solids, negative thermal expansion (NTE) has attracted enormous attention over the years. ${ }^{1-3}$ These unusual materials, in which the volume (of unit cell) contracts upon heating, are fundamentally interesting and technologically important. To date, varieties of compounds including framework compounds, ${ }^{1,4}$ ferroelectrics,${ }^{5}$ magnetics, ${ }^{6,7}$ and those with a valence change have been revealed with NTE features. ${ }^{8}$ By utilizing NTE materials to form either a single phase or a composite, the controllable coefficient of thermal expansion (CTE) of solids is highly expected which benefits equipment working at variable temperatures to reduce thermal failure. Moreover, smart materials or sensors could be realized through the interplay between elastic and physical properties during the unusual NTE progress in functional materials such as ferroelectrics and magnetics.

Different models have been proposed to elucidate NTE in such kinds of materials. Low-frequency vibration modes with a negative Grüneisen parameter are vital to the NTE in framework structures such as $\mathrm{ZrW}_{2} \mathrm{O}_{8} \cdot{ }^{9}$ The magnetovolume effect (MVE) or the Invar effect is often triggered by $\Gamma_{5 \mathrm{~g}}$ magnetic transition. $^{6}$ The spontaneous volume ferroelectrostriction

\footnotetext{
${ }^{a}$ Department of Physical Chemistry, University of Science and Technology Beijing, Beijing 100083, China. E-mail: xing@ustb.edu.cn

${ }^{b}$ Department of Physics and State Key Laboratory of Low-Dimensional Quantum Physics, Tsinghua University, Beijing 100084, China

${ }^{c}$ Department of Chemistry and Chemical Engineering, University of Science and Technology Beijing, Beijing 100083, China

$\dagger$ Electronic supplementary information (ESI) available. See DOI: 10.1039/ c6cp00011h
}

(SVFS), a recently raised mechanism, is applied to the NTE in ferroelectrics. ${ }^{3}$ As being valuable to electronic technology, ferroelectrics showing NTE have been studied intensively in the past decade, however, only in $\mathrm{PbTiO}_{3}$-based perovskite-type compounds. Actually, the NTE is quite rare among prototypic ferroelectrics. Most ferroelectrics exhibit normal volumetric expansion, for example, $+2.8 \times 10^{-5} \mathrm{~K}^{-1}$ of $\mathrm{BaTiO}_{3}(\mathrm{P} 4 \mathrm{~mm})$ and $+3.8 \times$ $10^{-5} \mathrm{~K}^{-1}$ of $\mathrm{LiNbO}_{3}(R 3 c) .^{10,11}$ The unusual NTE in ferroelectrics was first found in perovskite-type $\mathrm{PbTiO}_{3}(\mathrm{P} 4 \mathrm{~mm})$ with an averaged volumetric CTE of $-2.0 \times 10^{-5} \mathrm{~K}^{-1}(300-763 \mathrm{~K}) .{ }^{12,13}$ Afterward, enhanced NTE was achieved by forming a solution with $\mathrm{BiMO}_{3}$ $\left(M=\mathrm{Fe}, \mathrm{Zn}_{1 / 3} \mathrm{Nb}_{2 / 3}\right){ }^{3}$ Other ferroelectrics, especially the nonperovskite and lead-free ones, have not been concerned to NTE studies yet.

It is known that the $\mathrm{Pb}$ (II) $6 \mathrm{~s}-\mathrm{O} 2 \mathrm{p}$ interaction is essential for the large distortion as well as the NTE in $\mathrm{PbTiO}_{3} \cdot{ }^{3,14}$ Besides, the divalent $\mathrm{Sn}$ (II) owns similar valence electronic configuration of $5 \mathrm{~s}$ and could be ferroactive with some appropriate anions. Instead of oxides, the chalcogenide $\mathrm{Sn}_{2} \mathrm{P}_{2} \mathrm{~S}_{6}$ is a proper ferroelectric. The unit cell of $\mathrm{Sn}_{2} \mathrm{P}_{2} \mathrm{~S}_{6}$ adopting monoclinic $P_{\mathrm{n}}$ symmetry builds with rigid $\left(\mathrm{P}_{2} \mathrm{~S}_{6}\right)$ octahedra and two nonequivalent $\left(\mathrm{SnS}_{8}\right)$ polyhedra (Fig. 1a). The $P_{\mathrm{S}}$ vector lies in an $a c$-plane with a major component along the $a$-axis and a minor one along the $c$-axis (none along unique $b$-axis). It displays a moderate spontaneous polarization $\left(P_{\mathrm{S}} \sim 14 \mu \mathrm{C} \mathrm{\textrm {cm } ^ { - 2 }}\right)$ at room temperature and transforms into the paraelectric phase $\left(P 2_{1} / n\right)$ at $T_{\mathrm{C}} \sim 339 \mathrm{~K}$ through a second-order phase transition. ${ }^{15}$ Axial thermal expansions of $\mathrm{Sn}_{2} \mathrm{P}_{2} \mathrm{~S}_{6}$ were once measured using a dilatometer with single crystals. ${ }^{16,17}$ However, the resulted axial CTEs look quite discrepant (Fig. S5, ESI $\dagger$ ) mainly because of the domain orientations in ferroelectric crystals, which act as an 

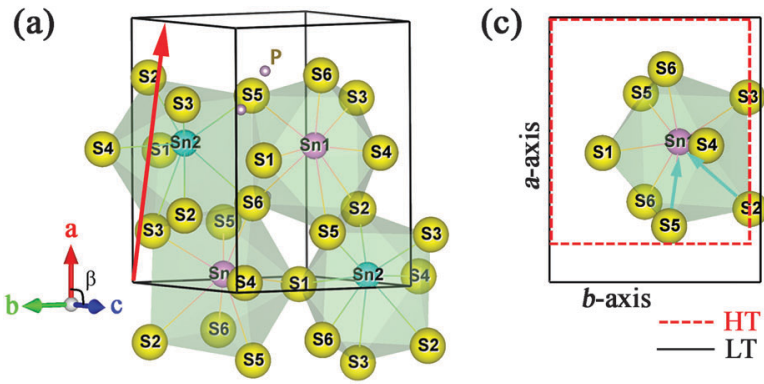

(b)

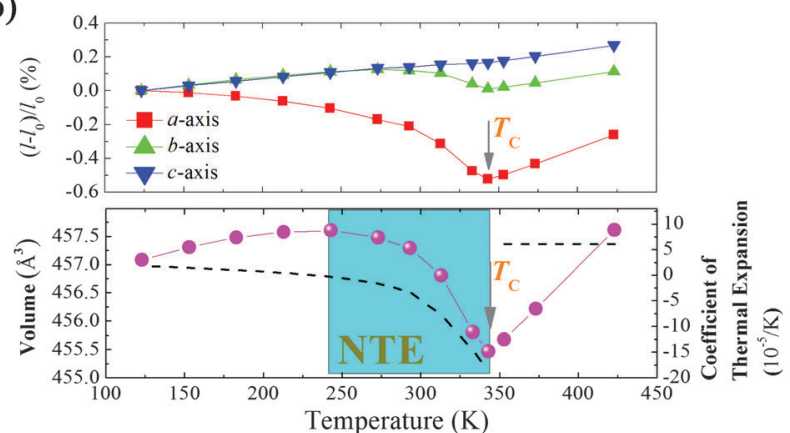

Fig. 1 (a) Unit cell and $P_{S}$ vector of ferroelectric $S n_{2} P_{2} S_{6}$. Short $P-P$ and $\mathrm{P}-\mathrm{S}$ bonds are omitted for clarity. (b) Evolutions of lattices and cell volume as a function of temperature (error bars are much smaller than labels). The volumetric coefficient of thermal expansion (CTE) is displayed with dashed lines. (c) Schematic of NTE in $\mathrm{Sn}_{2} \mathrm{P}_{2} \mathrm{~S}_{6}$. The scale of NTE is amplified. Arrows indicate intensely reduced $\mathrm{Sn} 1-\mathrm{S}$ distances as discussed below.

extrinsic effect involved in the dilatometric measurements. Moreover, the mechanism of the seeming NTE in $\mathrm{Sn}_{2} \mathrm{P}_{2} \mathrm{~S}_{6}$ is still unclear. In this work, we firstly reveal the nature of NTE in $\mathrm{Sn}_{2} \mathrm{P}_{2} \mathrm{~S}_{6}$ by determining the structure evolution and combining the first-principles calculations. Interestingly, the SVFS mechanism could well elucidate the NTE in $\mathrm{Sn}_{2} \mathrm{P}_{2} \mathrm{~S}_{6}$, which is the first NTE case in a non-perovskite lead-free ferroelectric material.

\section{Experimental}

The well crystalline $\mathrm{Sn}_{2} \mathrm{P}_{2} \mathrm{~S}_{6}$ powders with a grain size about $2 \mu \mathrm{m}$ (Fig. S1, ESI $\dagger$ ) were prepared in an evacuated sealed quartz tube. ${ }^{18}$ The ferroelectric-paraelectric phase transition temperature was confirmed to be $338 \mathrm{~K}$ using Differential Scanning Calorimetry (DSC) (Fig. S2, ESI $\dagger$ ). The Raman spectrum was also carried out to verify the phase composition of the as-synthesized powders (Fig. S3, ESI $\dagger$ ). Characterization of thermal expansion was performed using a high-resolution Panalytical X'Pert Powder X-ray Diffractometer (XRD, Cu K $\alpha$ ). Full patterns at non-ambient temperatures were collected in an Anton-Paar TTK450 chamber from $123 \mathrm{~K}$ to $423 \mathrm{~K}$ with a 10-30 K interval, and then conducted the Rietveld refinements with FULLPROF. ${ }^{19}$ Afterward, the effects of the Sn(II)-S interaction on both spontaneous polarization $\left(P_{\mathrm{S}}\right)$ and NTE are revealed using the first-principles calculations. The details of experiments, structure refinements and calculation processes are available in the ESI. $\dagger$

\section{Results and discussion}

The obtained full XRD patterns were well refined using the starting structure model (ICSD-25357) reported by G. Dittmar (Fig. S4, ESI $\dagger$ ). The typical $R_{\mathrm{wp}}$ is below 0.05 and the accurate lattice parameters are obtained (Table S1, ESI $\dagger$ ). As shown in Fig. $1 \mathrm{~b}$, the evolution routes of $a$-, $b$-axis change abruptly at around $343 \mathrm{~K}$, the temperature near the $T_{\mathrm{C}}$. The major polar $a$-axis displays continuous contraction below $T_{\mathrm{C}}$ while the minor polar $c$-axis shows normal expansion. Another contraction along the non-polar $b$-axis is also observed from $273 \mathrm{~K}$ to $T_{\mathrm{C}}$. In particular, the cell volume displays a remarkable NTE from $243 \mathrm{~K}$ to $T_{\mathrm{C}}$ and a minimum value at $T_{\mathrm{C}}$. The averaged volumetric CTE is as large as $-4.7 \times 10^{-5} \mathrm{~K}^{-1}$ within an appropriate $100 \mathrm{~K}$ temperature interval. Note that the strength of this NTE is competitive to that of several popular magnetics, such as $-3.3 \times 10^{-5} \mathrm{~K}^{-1}$ of $\mathrm{LaFe}_{10.6} \mathrm{Si}_{2.4}(260-370 \mathrm{~K})$ and $-3.6 \times$ $10^{-5} \mathrm{~K}^{-1}$ of $\mathrm{Mn}_{3} \mathrm{Cu}_{0.5} \mathrm{Ge}_{0.5} \mathrm{~N}(280-365 \mathrm{~K}),{ }^{6,7}$ and also the superior one among ferroelectrics, $-3.9 \times 10^{-5} \mathrm{~K}^{-1}$ of $0.4 \mathrm{PbTiO}_{3}-$ $0.6 \mathrm{BiFeO}_{3}(300-950 \mathrm{~K}){ }^{3}{ }^{3}$ Besides the unusual NTE, the CTE of $\mathrm{Sn}_{2} \mathrm{P}_{2} \mathrm{~S}_{6}$ below $243 \mathrm{~K}$ is as low as $+0.94 \times 10^{-5} \mathrm{~K}^{-1}$. In contrast, a robust one, $+5.90 \times 10^{-5} \mathrm{~K}^{-1}$ appears in the paraelectric phase. Thus, the abnormally low CTE and NTE of volume below $T_{\mathrm{C}}$ must result from an additionally negative contribution relating to the spontaneous polarization $\left(P_{\mathrm{S}}\right)$ of the ferroelectric phase. Without ferroelectricity, the paraelectric phase expands as common materials due to the anharmonic atomic vibration.

It is known that the appearance of $P_{\mathrm{S}}$ in $\mathrm{Sn}_{2} \mathrm{P}_{2} \mathrm{~S}_{6}$ accompanies the relative displacements of Sn(II) cations to the charge center of the $\mathrm{S}_{8}$ polyhedron, i.e., $P_{\mathrm{S}}$ displacement $\left(\delta_{\mathrm{Sn}}\right)$, which could be acquired from a refined structure. The calculated major $\delta_{\mathrm{Sn}}$ (along $a$-axis) at $293 \mathrm{~K}$ is $0.19 \AA$ at the $\mathrm{Sn} 1$ site and $0.32 \AA$ at the Sn2 site, close to the reported values $(0.22 \AA$, $0.30 \AA$ ) of single crystals. ${ }^{20}$ Fig. 2a displays the temperature dependent major $-\delta_{\mathrm{Sn}}$. Both decrease upon heating and the one at the Sn1 site weakens much more considerably than that at the $\mathrm{Sn} 2$ site. In $\mathrm{Sn}_{2} \mathrm{P}_{2} \mathrm{~S}_{6}$ the hybridization between $\mathrm{Sn} 5 \mathrm{~s}$ and $\mathrm{S} 3 \mathrm{p}$ is suggested to be crucial to ferroelectricity. ${ }^{21}$ To further investigate the above behaviors of $\delta_{\mathrm{Sn}}$ and its correlation with NTE in $\mathrm{Sn}_{2} \mathrm{P}_{2} \mathrm{~S}_{6}$, first-principles calculations were performed to explore the varying $\mathrm{Sn}-\mathrm{S}$ bonding character in the ferroelectric phase using the Vienna ab-initio simulation package (VASP). Several refined structures at $123 \mathrm{~K}, 273 \mathrm{~K}$, and $313 \mathrm{~K}$ were calculated respectively without further optimization. The one at $373 \mathrm{~K}$ was also included to examine the case in the paraelectric phase.

As shown in Fig. 2b, the Sn $5 \mathrm{~s}$ states hybridize with the $\mathrm{S} 3 \mathrm{p}$ states in several energy intervals, especially from -7.4 to $-6.2 \mathrm{eV}$. The electronic distribution of these states clearly displays hybridization in several Sn-S bonds (Fig. S6, ESI†). Similar to the result of the $\mathrm{Pb}$ (II) $6 \mathrm{~s}-\mathrm{O} 2 \mathrm{p}$ interaction, ${ }^{22}$ only a small amount of $\mathrm{Sn} 5 \mathrm{~s}$ states locates at the top of the valence band where the major $\mathrm{S} 3 \mathrm{p}$ states occupy. These top $5 \mathrm{~s}$ states should be caused by $5 \mathrm{~s}-3 \mathrm{p}$ mixing and relate to the antibonding states. The electrons calculated corresponding to the valence band maximum (VBM) show asymmetrical shapes around $\mathrm{Sn}(\mathrm{II})$ cations (Fig. 2c), pointing to these far sulfur anions and appropriately paralleling to the 
(a)

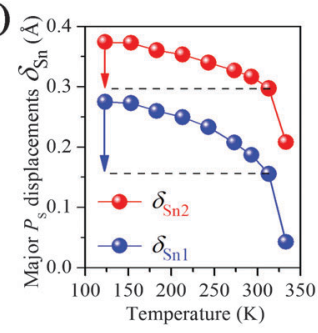

(b)

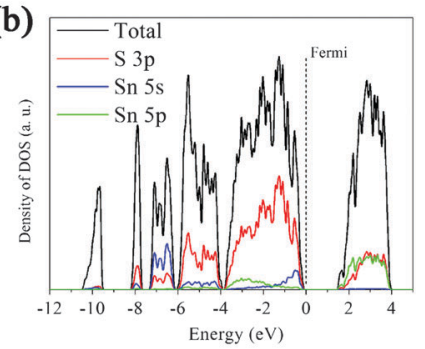

(c)

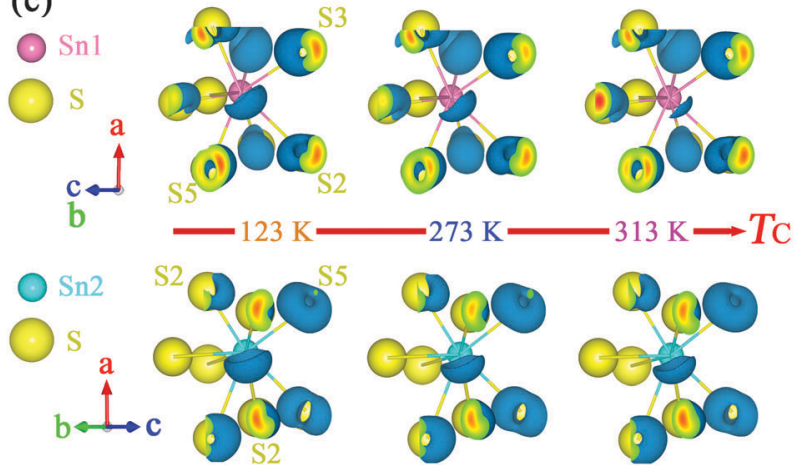

Fig. 2 (a) Temperature dependent major $P_{\mathrm{S}}$ displacements $\left(\delta_{\mathrm{Sn}}\right)$ at two non-equivalent $\mathrm{Sn}(\mathrm{I})$ sites. The maximum error is $0.014 \AA$ for $\delta_{\mathrm{Sn}}$ at $333 \mathrm{~K}$, whose size is close to the circle label. (b) Calculated total DOS and PDOS of Sn $5 s, 5 p$ and S $3 p$ according to the structure at $273 \mathrm{~K}$. (c) Evolution of the asymmetrical electrons concentrating around $\mathrm{Sn}(I)$ sites below $T_{\mathrm{C}}$. The isosurface of electron density is 0.0270 e $\AA^{-3}$.

polar $a$-axis. This brings to mind the lone pair electrons (LPEs) exhibiting stereochemical activity. Our results coincide more with the revised concept of LPEs, in which it is the direct cation-anion interaction rather than the $5 \mathrm{~s} / 6 \mathrm{~s}$ electrons that induces structure distortion. ${ }^{22}$ Here, we still refer to these hat-like electrons as LPEs. As the structure changes from $123 \mathrm{~K}$ to $313 \mathrm{~K}$, the bonding states weaken slightly and similarly at two Sn(II) sites (Fig. S6, ESI $\dagger$ ). Interestingly, these LPEs disappear markedly at the Sn1 site while not at the Sn2 site, matching well with the distinct evolution of $P_{\mathrm{S}}$ displacements as discussed. In the paraelectric structure, none of the LPEs could be seen around the Sn(II) cation (Fig. S7, ESI $\dagger$ ). Thus the LPEs induced by the $\mathrm{Sn}$ (II)-S interaction contribute essentially to the local distortion and favor the appearance of ferroelectricity.

After the above studies, we can speculate how this NTE could happen. The $\mathrm{Sn}$ (II)-S interaction in the ferroelectric phase gives rise to anisotropic Sn-S bonding, i.e., short covalent and long ionic bonds (Fig. S6, ESI $\dagger$ ). With rising temperature, the $\mathrm{Sn}(\mathrm{II})-\mathrm{S}$ interaction is depressed by thermal vibrations and then the LPEs concentrating around the Sn(II) site become smaller until the phase transition at $T_{\mathrm{C}}$. The electronic repulsion between these electrons and front sulfur anions is thus reduced, then the elongated $\mathrm{Sn}-\mathrm{S}$ bonds are released, allowing those distant anions to move backward to the Sn(II) cations. This progress permits the contraction of these long Sn-S distances, which may lead to the NTE in terms of the crystal structure. Fig. 3 displays the temperature dependent bond lengths of $\mathrm{Sn}-\mathrm{S}$ as a result of structure refinements. The two long bonds around the

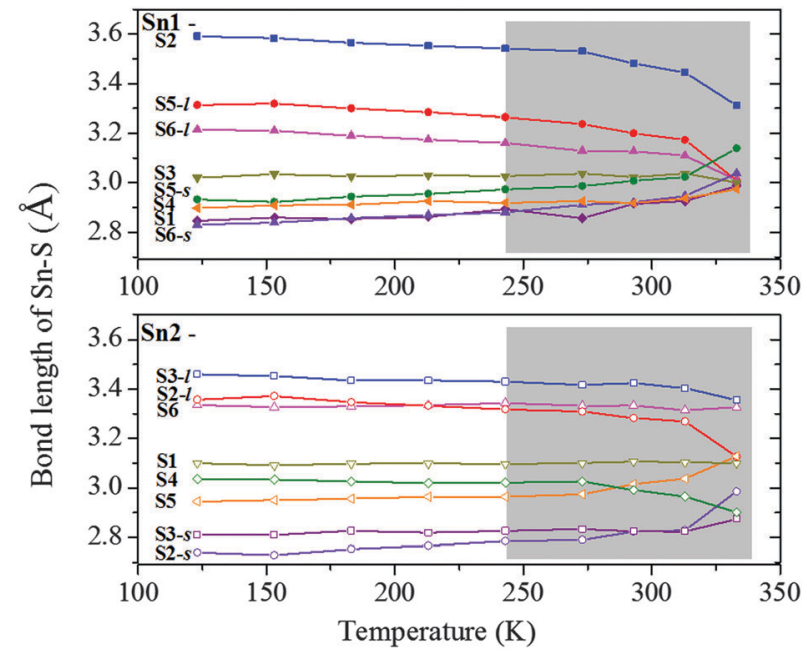

Fig. 3 Temperature dependent bond lengths of $\mathrm{Sn}-\mathrm{S}$ in $\mathrm{Sn}_{2} \mathrm{P}_{2} \mathrm{~S}_{6}$. The errors are less than $0.01 \AA$, smaller than the labels. The symbols "-s" and "-l" denote short covalent and long ionic Sn-S bonds respectively. The shadow regions indicate where the NTE occurs.

Sn1 site (Sn1-S2 \& Sn1-S5- $l$ ) decrease much more rapidly than the ones around the Sn2 site (Sn2-S3-l \& Sn2-S6), especially during the NTE region. This is consistent with the distinct evolution of LPEs at two Sn(II) sites. Moreover, the contraction of long ionic bonds around the Sn1 site looks severe than the elongation of short covalent ones. From 243 to $333 \mathrm{~K}$, the long Sn1-S bonds contract by $0.25,0.23$ and $0.15 \AA$ (7.6\%, $6.5 \%$ and $4.7 \%$ ), while the short ones elongate mostly by $0.17,0.16$ and $0.10 \AA(5.7 \%, 5.6 \%$ and $3.4 \%)$, respectively. The contractions exceed the elongations by about $0.05 \AA$ A. Note that the contraction of the polar $a$-axis in this region is $0.035 \AA$, comparable with $0.05 \AA$ A. Thus we suggest that the asymmetric change of Sn1-S bonds finally triggers the NTE. Fig. 1c illuminates the progress of NTE in $\mathrm{Sn}_{2} \mathrm{P}_{2} \mathrm{~S}_{6}$.

The NTE in $\mathrm{Sn}_{2} \mathrm{P}_{2} \mathrm{~S}_{6}$ was once ascribed to the negative Grüneisen coefficients of optical vibrations ( $\gamma_{\text {optic }}$ ) as the case in $\mathrm{ZrW}_{2} \mathrm{O}_{8}$. Based on the thermal expansion behavior, the calculated $\gamma_{\text {optic }}$ changes its sign around $200 \mathrm{~K}$ and gets more and more negative until the phase transition. ${ }^{23}$ According to the relation $k_{\mathrm{B}} T=\hbar \omega_{\mathrm{i}}$, where $k_{\mathrm{B}}$ is the Boltzmann constant and the $\hbar$ is the reduced Planck constant, the modes accounting for the NTE should have approximate frequencies around $140 \mathrm{~cm}^{-1}$. However, high-pressure Raman studies of $\mathrm{Sn}_{2} \mathrm{P}_{2} \mathrm{~S}_{6}$ show that almost all optical modes above $150 \mathrm{~cm}^{-1}$ present positive $\gamma_{\text {optic }}$ values. ${ }^{24}$ Moreover, with increasing temperature, the weighted $\gamma_{\text {optic }}$ should be more positive instead of being more negative since high-frequency optical vibrations tend to be active. Thus, the thermodynamic calculation conflicts with the experimental results. Therefore, the NTE in ferroelectric $\mathrm{Sn}_{2} \mathrm{P}_{2} \mathrm{~S}_{6}$ is difficult to be described simply through the Grüneisen model.

As a new case in non-perovskite lead-free ferroelectrics, it is necessary to further examine the correlation of NTE with the ferroelectricity in $\mathrm{Sn}_{2} \mathrm{P}_{2} \mathrm{~S}_{6}$ and see whether it is compatible to the SVFS mechanism. The contribution of $P_{\mathrm{S}}$ to the volume expansion in $\mathrm{Sn}_{2} \mathrm{P}_{2} \mathrm{~S}_{6}, \omega_{\mathrm{s}}$ is calculated as $\left(V_{\exp }-V_{\mathrm{nm}}\right) / V_{\mathrm{nm}} \times 100 \%$, 
(a)
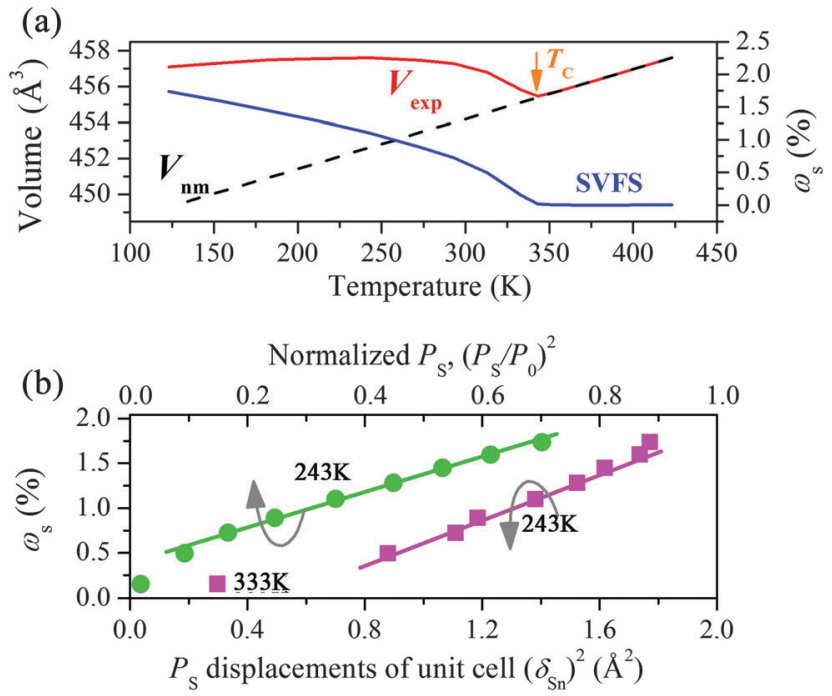

Fig. 4 (a) Temperature dependent $V_{\exp }, V_{n m}$ and magnitude of negative contribution to thermal expansion from ferroelectricity, $\omega_{\mathrm{s}}$. (b) Linear correlations of $\omega_{\mathrm{S}} \sim\left(P_{\mathrm{S}} / P_{0}\right)^{2}$ and $\omega_{\mathrm{S}} \sim\left(\delta_{\mathrm{Sn}}\right)^{2} . \delta_{\mathrm{Sn}}$ here is the total $P_{\mathrm{S}}$ displacement of $\mathrm{Sn}(I)$ cations in the single unit cell. The maximum error of $\left(\delta_{\mathrm{Sn}}\right)^{2}$ is $0.02 \AA^{2}$ at $333 \mathrm{~K}$, smaller than the square label.

in which $V_{\text {exp }}$ is the observed volume and $V_{\mathrm{nm}}$ is a nominal one extrapolated from the paraelectric phase (Fig. 4a). Then $\omega_{\mathrm{s}}$ is plotted as the square of polarization as shown in Fig. $4 \mathrm{~b}$. It is unambiguous that the $\omega_{\mathrm{s}}$ correlates strongly with both the squared $P_{\mathrm{S}}$ displacement, $\left(\delta_{\mathrm{Sn}}\right)^{2}$ and the $\left(P_{\mathrm{S}} / P_{0}\right)^{2}$ (normalized $P_{\mathrm{S}}$ from literature data of $\mathrm{Sn}_{2} \mathrm{P}_{2} \mathrm{~S}_{6}$ crystals in ref. 25$)$. The dominated linearity below $T_{\mathrm{C}}$ well suggests that the $P_{\mathrm{S}}$ correlates inherently with the $\omega_{\mathrm{s}}$ and governs the negative contribution to the cell volume. Hence, the SVFS mechanism is applicable to account for the NTE here. Actually, it is expected to see such a linearity between the ferroelectricity and the $\omega_{\mathrm{s}}$, since the LPEs impact the bond lengths and the $P_{\mathrm{S}}$ simultaneously as discussed above.

Though here the $P_{\mathrm{S}}$ is much inferior compared with the one in $\mathrm{PbTiO}_{3}\left(14\right.$ vs. $60 \mu \mathrm{C} \mathrm{cm}{ }^{-2}$ at $300 \mathrm{~K}$ ), the SVFS seems intense enough to trigger NTE in $\mathrm{Sn}_{2} \mathrm{P}_{2} \mathrm{~S}_{6}$. It is interesting to see both ferroelectrics, actually the sole two prototypic ones showing remarkable NTE, involve the $\mathrm{s}-\mathrm{p}$ interaction $(\mathrm{Pb}-\mathrm{O}$ and $\mathrm{Sn}-\mathrm{S})$ to generate the $P_{\mathrm{S}}$. This well indicates the special role of this interaction and the LPEs in the NTE behavior of ferroelectrics, which is worthy of more studies. In addition, besides the NTE behavior, $\mathrm{Sn}_{2} \mathrm{P}_{2} \mathrm{~S}_{6}$ also displays semi-conductive character and could be easily grown into large crystals. These features, which are absent in $\mathrm{PbTiO}_{3}$, permit more possibilities to design special materials or devices with the unusual NTE behavior.

\section{Conclusions}

In conclusion, the unusual negative thermal expansion in nonperovskite lead-free ferroelectric $\mathrm{Sn}_{2} \mathrm{P}_{2} \mathrm{~S}_{6}$ is carefully studied. A large NTE of $-4.7 \times 10^{-5} \mathrm{~K}^{-1}$ is determined from $243 \mathrm{~K}$ to $T_{\mathrm{C}}(338 \mathrm{~K})$. The evolution of $P_{\mathrm{S}}$ displacement and NTE progress are clarified by means of structure analyses and first-principles calculations.
Then the NTE mechanism of spontaneous volume ferroelectrostriction (SVFS), which is developed from perovskite-type leadbased ferroelectrics, is firstly extended to the elucidation of NTE in non-perovskite and lead-free ferroelectric $\mathrm{Sn}_{2} \mathrm{P}_{2} \mathrm{~S}_{6}$. In addition, it is further suggested that $s-p$ interaction and LPEs play a significant role in obtaining remarkable NTE in ferroelectrics. This work will considerably motivate the design of new ferroelectric and functional NTE materials.

\section{Acknowledgements}

This work is supported by NSFC (91022016, 21031005, 21590793, 21231001, 91422301), Program for Changjiang Scholars (IRT1207) and Funds for the Central Universities, China (Grant No. FRF-SD-13-008A).

\section{Notes and references}

1 T. A. Mary, J. S. O. Evans, T. Vogt and A. W. Sleight, Science, 1996, 272, 90-92.

2 K. Takenaka, Sci. Technol. Adv. Mater., 2012, 13, 013001.

3 J. Chen, L. Hu, J. Deng and X. Xing, Chem. Soc. Rev., 2015, 44, 3522-3567.

4 B. K. Greve, K. L. Martin, P. L. Lee, P. J. Chupas, K. W. Chapman and A. P. Wilkinson, J. Am. Chem. Soc., 2010, 132, 15496-15498.

5 J. Chen, K. Nittala, J. S. Forrester, J. L. Jones, J. Deng, R. Yu and X. Xing, J. Am. Chem. Soc., 2011, 133, 11114-11117.

6 K. Takenaka and H. Takagi, Appl. Phys. Lett., 2005, 87, 261902.

7 R. Huang, Y. Liu, W. Fan, J. Tan, F. Xiao, L. Qian and L. Li, J. Am. Chem. Soc., 2013, 135, 11469-11472.

8 M. Azuma, W. Chen, H. Seki, M. Czapski, S. Olga, K. Oka, M. Mizumaki, T. Watanuki, N. Ishimatsu, N. Kawamura, S. Ishiwata, M. G. Tucker, Y. Shimakawa and J. P. Attfield, Nat. Commun., 2011, 2, 347.

9 R. Mittal, S. L. Chaplot, H. Schober and T. A. Mary, Phys. Rev. Lett., 2001, 86, 4692-4695.

10 H. E. Kay and P. Vousden, Philos. Mag., 1949, 40, 1019.

11 S. C. Abrahams, H. J. Levinstein and J. M. Reddy, J. Phys. Chem. Solids, 1966, 27, 1019-1026.

12 G. Shirane and H. Sadao, J. Phys. Soc. Jpn., 1951, 6, 265-270.

13 X. Xing, J. Deng, J. Chen and G. Liu, Rare Met., 2003, 22, $1-4$.

14 Y. Kuroiwa, S. Aoyagi, A. Sawada, J. Harada, E. Nishibori, M. Takata and M. Sakata, Phys. Rev. Lett., 2001, 87, 217601.

15 C. D. Carpentier and R. Nitsche, Mater. Res. Bull., 1974, 9, 1097-1100.

16 A. Say, O. Mys, A. Grabar, Y. M. Vysochanskii and R. Vlokh, Phase Transitions, 2009, 82, 531-540.

17 Y. M. Vysochanskii, T. Janssen, R. Currat, R. Folk, J. Banys, J. Grigas and V. Samulionis, Phase transitions in phosphorous chalcogenide ferroelectrics, Vilnius University Publishing House, Vilnius, 2008. 
18 I. N. Rybina, T. G. Lupeiko, S. N. Svirskaya and A. A. Nesterov, Inorg. Mater., 2010, 46, 1242-1243.

$19 \mathrm{~J}$. Rodriguez-Carvajal, Abstracts of the Satellite Meeting on Powder Diffraction of the XV Congress of the IUCr, Toulouse, France, 1990, p. 127.

20 B. Scott, M. Pressprich, R. D. Willet and D. A. Cleary, J. Solid State Chem., 1992, 96, 294-300.

21 K. Glukhov, K. Fedyo, J. Banys and Y. M. Vysochanskii, Int. J. Mol. Sci., 2012, 13, 14356-14384.
22 D. J. Payne, R. G. Egdell, A. Walsh, G. W. Watson, J. Guo, P.-A. Glans, T. Learmonth and K. E. Smith, Phys. Rev. Lett., 2006, 96, 157403.

23 Y. Vysochanskii, A. Kohutych, R. Yevych and S. Perechinskii, AIP Conf. Proc., 2009, 209, 209-215.

24 V. V. Shchennikov, N. V. Morozova, I. Tyagur, Y. Tyagur and S. V. Ovsyannikov, Appl. Phys. Lett., 2011, 99, 212104.

25 R. M. Yevych and Y. M. Vysochanskii, Condens. Matter Phys., 2008, 11, 417-423. 